\title{
Matrix metalloproteinases as potential fecal biomarkers for ulcerative colitis - a function beyond their proteolytic activity
}

This article was published in the following Dove Press journal:

Metalloproteinases In Medicine

27 May 2016

Number of times this article has been viewed

\author{
Adani Pujada' \\ Lewins Walter ${ }^{2}$ \\ Tanvi Dhere ${ }^{3}$ \\ Pallavi Garg' \\ 'Department of Biology, ${ }^{2}$ Center for \\ Inflammation, Immunity, and Infection, \\ Institute of Biomedical Sciences, \\ Georgia State University, ${ }^{3}$ Division of \\ Digestive Diseases, Emory University, \\ Atlanta, GA, USA
}

\begin{abstract}
Specific, sensitive, and noninvasive biomarkers are the most relevant tools to monitor activity, early stage detection, and the treatment management of ulcerative colitis (UC). Although, there is no single "Gold Standard" test for the diagnosis of UC, clinicians utilize a combination of clinical score, histological score, and colonoscopy index to assess disease activity and to predict the outcome of the disease. Over a decade, different biomarkers from body fluids have been considered to assess UC. The disadvantage of these biomarkers (as for example, serum or blood) is that they can be influenced by other inflammatory diseases. Feces formation mainly occurs in colon; therefore, fecal biomarkers could be the most sensitive and specific ones for UC assessment compared to any other body-fluid biomarkers. Fecal biomarkers are the proteins that either secreted out or are generated by the inflamed intestinal mucosa. The main advantage of them is their use in diagnosing and assessing disease activity in difficult cases, especially when invasive strategies can be very stressful for the patients. In this review, we validate a panel of matrix metalloproteinases (MMPs) as fecal biomarkers demonstrating not only their capacity as inflammatory markers but also their specificity to colonic inflammation. Based on our literature search, we also identify MMP9 as the most promising fecal biomarker for UC activity.
\end{abstract}

Keywords: Cytokines, calprotectin, lipocalin, inflammatory bowel disease, UC therapy, MMP1, MMP3, MMP7, MMP10, MMP14

\section{Introduction}

Inflammatory bowel disease (IBD) is a broad term that describes conditions with chronic or recurring immune response and inflammation of the gastrointestinal (GI) tract. The two most common IBDs are ulcerative colitis (UC) and Crohn's disease (CD). The human matrix metalloproteinases (MMPs) are a family of 24 zinc-dependent endopeptidases. ${ }^{1}$ There is mounting evidence that MMPs are the predominant proteases in gut mucosa during active IBD and play an important role in ulceration and tissue remodeling. ${ }^{2,3}$ They are known to be upregulated in IBD and other inflammatory conditions by degrading extracellular matrix and proteolytic cleavage of bioactive proteins such as cytokines, chemokines, receptors, proteases, and adhesion molecules. ${ }^{3,4}$

IBD is a complex disease that arises as a result of the interaction of environmental and genetic factors; hence, it is a substantial public health problem. In this scenario, efficient assessments and early prognosis are not only important but also very challenging. The most common approach for clinical evaluations is colonoscopy, complemented with blood markers and/or serological markers..$^{5}$ Although colonoscopy is a gold standard for monitoring disease activity, ${ }^{6}$ it needs a good communication between gastroenterologist and histopathologist for the interpretation of the colonoscopy
Correspondence: Pallavi Garg

Department of Biology, Georgia State University, 100 Piedmont Avenue SE, Petit Science Center, Room 516,Atlanta, GA, USA

Tel + I 4044135416

Fax +I 4044133580

Email pgarg@gsu.edu
Metalloproteinases In Medicine 2016:3 19-29

(c) (1) (5) 2016 Pujada et al. This work is published and licensed by Dove Medical Press Limited. The full terms of this license are available at https://www.dovepress.com/terms. (c) 1 BY ${ }_{\mathrm{NC}}$ php and incorporate the Creative Commons Attribution - Non Commercial (unported, v3.0) License (http://creativecommons.org/licenses/by-nc/3.0/). By accessing the work you hereby accept the Terms. Non-commercial uses of the work are permitted without any further permission from Dove Medical Press Limited, provided the work is properly attributed. For permission for commercial use of this work, please see paragraphs 4.2 and 5 of our Terms (https.//www.dovepress.com/terms.php).
Dovepress

http://dx.doi.org/10.2147/MNM.S52956

19

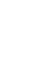

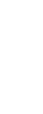


assessment. ${ }^{7}$ Additionally, colonoscopy might fail to detect early inflammatory stages or localized inflammatory areas. ${ }^{8}$ On the other hand, blood markers or serum markers can be elevated under any inflammatory conditions, and therefore cannot be significantly specific for IBD assessment. The significance of a biomarker is to gain an objective measurement of disease activity (as symptoms are often subjective) and being a noninvasive procedure (unlike colonoscopy). In this review, we discuss fecal MMPs (especially MMP9) as a new diagnostic tool for measuring colonic inflammation (UC in particular). Fecal markers fulfill all the criteria of being noninvasive, simple, cost-effective, as well as specific and sensitive for assessing colonic inflammation. ${ }^{5,8}$

\section{Crohn's disease}

Although both CD and UC share similar symptoms, there are remarkable differences in their location as well as their progression in the GI tract. CD affects anywhere from mouth to anus. According to recent studies, the prevalence of CD ranges from 100 to 200 cases/100,000 people annually in the US. ${ }^{9}$ The primary symptoms of CD include continuous diarrhea, fever, and weight loss. ${ }^{10,11} \mathrm{CD}$ is characterized by the patchy lesions throughout the GI tract, specifically in both small and large intestines, including the most affected regions as terminal ileum and/or the perianal regions. ${ }^{3} \mathrm{CD}$ penetrates the deep layers of the intestine; therefore, transmural inflammations are common among CD-affected patients and prolong exposure causes ulcerations. ${ }^{10,11} \mathrm{CD}$-associated complications can lead to intestinal blockage, bleeding intestine, perforation of the bowel, fistula, abscess, and toxic megacolon. Chronic CD patients are at an increased risk of developing small-bowel cancer, colon cancer, and colorectal cancer (CRC). ${ }^{12-14}$

Although the etiology for CD is unknown, recent research suggests that in addition to genetic and environmental factors, imbalance of the gut microbiota also contributes to its development. ${ }^{15}$ Commensal bacteria in the host intestine epithelial monolayer are diffused through mucosal layers and are able to perform or aid in multiple important functions such as digestion, metabolizing nutrients, preventing infections caused by invasion of foreign pathogens, and maintaining the homeostasis of mucosal immune system. In $\mathrm{CD}$, both the epithelial monolayer and the mucosal layer are disrupted, inducing an imbalance in gut microbiota population. This results in a decrease of intestinal protective bacterial population such as Lactobacillus and an increase in harmful intestinal bacterial population such as Escherichia coli. Thus, the balance between host microbiota and invading pathogens plays an important role and is very critical in the development of CD and is favored in genetically susceptible host. Furthermore, different biological pathways such as autophagy, apoptosis, and necrosis can also play a role in the development and the maintenance of the intestinal bacteria balance in patients with $\mathrm{CD} .{ }^{13}$

The ulcerations caused by CD can be mediated by different pathways resulting in a large amount of proinflammatory cytokines, chemokines, reactive oxygen radicals, and metalloproteinases, suggesting their participation in the development of the inflammatory response to CD. ${ }^{15,16}$ Recent studies suggest that a large range of cell types secreting MMPs during CD reflects a higher correlation to the activity of this form of IBD. The higher concentration of these MMPs is due to cytokines such as tumor necrosis factor alpha (TNF $\alpha$ ), interleukin-1 beta (IL-1 $\beta$ ), and interferon gamma that are secreted by monocytes, neutrophils, and lymphocytes. Over the years, histological investigations based on tissues obtained from affected or CD inflamed region samples showed prominent and consistent upregulations of MMP1, MMP2, MMP3, and MMP9. ${ }^{15-18}$ It has been observed that MMP1 degrades insulin-like growth factor binding protein, thus releasing IGF; and MMP3 inactivates $\alpha 2$-antiplasmin, thus favoring local plasmin-mediated proteolysis during CD. MMP9 potentiates proinflammatory interleukin-8 (IL-8) by removing the first six amino-terminal amino acids and processes IL-1 into an active form. Both MMP2 and MMP9 degrade substance P, an important neurokine, and are associated with increased levels of the angiogenic factor, vascular endothelial growth factor, and factor VIII-related antigen. ${ }^{19}$ Overall, this suggests that MMPs play an important role in $\mathrm{CD}$ as key regulators of cell function by maintaining the intestinal homeostasis, remodeling the structural damage of tissue, and propagating the excessive immune response. ${ }^{16}$

\section{Ulcerative colitis}

$\mathrm{UC}$ is a form of IBD causing inflammation and ulcers in the colon either in segments or completely. The main symptom of active disease is usually constant diarrhea mixed with blood. UC, as its name suggests, affects only the colon and rectum, leaving the rest of the GI tract unscathed, and hence, it is very different from CD (another form of IBD). Although it has no known cause, presumed genetic component and susceptibility to western diet and lifestyle increases the prevalence of this disease. The disease may be triggered in a susceptible person by environmental factors, although dietary modifications may reduce the discomfort of a person with the disease. Recent studies of migrant populations as well as populations 
of developing countries have demonstrated a slow but steady increase in the incidence of UC. ${ }^{8}$ The incidence of UC is higher than that of CD in USA and several areas of Europe (except in Canada). Interestingly, the occurrence of UC preceded that of $\mathrm{CD}$ in the countries with rare incidences, such as South Korea, People's Republic of China, India, Iran, Lebanon, Thailand, the French West Indies, and North Africa. Prevalence of UC has increased in the past 50 years, up to 120-200/100,000 persons.

Epidemiologic studies of migrant populations indicate that genetic and environmental factors interact to determine risk for UC. Environmental factors include mainly the modern lifestyle: improved home amenities, widespread use of antibiotics, consumption of fat- and protein-rich diets, refrigeration, and industrial pollution.

UC occurs in the top layers of the colon and inflammatory lesions are generally diffuse and superficial, while deep ulcerations are observed only in patients with severe disease. ${ }^{8}$ Interestingly, in recent years, an increase in mortality is observed among newly diagnosed patients as well as patients with extensive disease..$^{20,21} \mathrm{UC}$ often develops complications such as toxic megacolon, massive colonic hemorrhage, strictures, fistulas, and abscesses. ${ }^{22,23}$ Prolong-term UC involves an increased risk of liver disease and CRC. ${ }^{22}$ Increased mortality is observed in the perioperative period in patients with severe disease, which contributes to increased death rate among newly diagnosed patients. Furthermore, with the prolong exposure, there is an increased risk of dying from UC-related causes such as liver disease and CRC.

Colonic epithelium has different protective mechanisms against inflammatory stimulations and important ones among those are elaboration and secretion of mucus through goblet cells. UC-associated inflammation arises due to imbalance between the environment, the immune system, and the associated susceptible genes and remains confined to the mucosal portion of the colon. Further, feces analyses make it easy to assess the host microbiome and the ingested virus, bacteria, or fungi, which play crucial roles by triggering inflammation directly or indirectly through immune dysregulation, ${ }^{24}$ and therefore could be good resource for efficient biomarkers. These microorganisms execute different important cellular functions such as protecting from invading pathogens, assisting in digestion and releasing nutrients, as well as maintaining the colonic immunity. ${ }^{13}$ Moreover, gut microbial products also can initiate inflammation as well as regulate immune cells and immune response in colonic epithelium. Hence, the dysregulation of immune response to enteric bacteria induces sustained, overproduction of multiple inflammatory cytokines and reactive oxygen and nitrogen species that directly or indirectly damage colonic tissues, leading to erosions, ulcerations fibrosis, and edema. ${ }^{25}$

\section{Matrix metalloproteinases}

MMPs are a large family of calcium-dependent, zinccontaining endopeptidases. They are classified mainly based on their substrate specificity (collagenases, gelatinases, stromelysins, matrilysins) and membrane type, while their MMP number has been assigned in order of discovery. ${ }^{26}$ Their expressions are regulated at different checkpoints, including transcriptional regulation, activation of the proenzymes, and the binding to endogenous tissue inhibitors (called tissue inhibitors of MMPs). ${ }^{27,28}$ Initially, they have been considered to be involved mainly with tissue remodeling and degradation of extracellular matrix. Although over a period of time, their multifaceted roles at almost all the junctures of inflammation as well as cancer have been recognized. ${ }^{29}$ MMPs uniquely modulate the biological activity of the first set of molecules that are released with the onset of inflammation, namely, cytokines, chemokines and serine proteases. MMPs are also involved in the adhesion and migration of leukocytes from blood vessels to the site of inflammation during the signaling cascade as an inflammatory response.

Secretion of MMPs is important for facilitating tissue damage as well as wound healing or tissue repair during IBD. ${ }^{2,3,30,31}$ In UC, ulceration of mucosa can be mediated by different pathways, resulting in large influx of neutrophils ${ }^{27}$ and marked by the secretion of neutrophil collagenase (MMP8). UC is also associated with upregulation of MMPs secreted by macrophages and fibroblasts, MMP1, MMP2, MMP3, MMP7, MMP9, MMP10, MMP12, and MMP14,27 in particular.

MMPs are well-established proteins associated with colonic inflammation, ${ }^{2,27}$ and most of them are mainly secreted by epithelial cells or neutrophil in the colonic lumen; hence, they can be considered as promising fecal biomarkers for UC. Many of these MMPs are expressed by inflammatory cells; however, in inflammation during tissue damage and repair, they are also secreted by epithelial cells and stromal cells. ${ }^{32}$ In general, human feces are a mixture of water, undigested food, microorganisms, and epithelial cells' shedding of GI tract. ${ }^{33}$ In the setting of inflammation, GI tract homeostasis is dysregulated and colon plays an important role in fecal formation; hence, colonic inflammation will be reflected in fecal composition. Moreover, during inflammation, feces will also have neutrophil-derived proteins, lymphocytes, and plasma cells. These substances do not degrade in feces 
and hence can be a measure of colonic inflammation or UC as biomarkers. ${ }^{8,34}$

\section{MMPI (collagenase I)}

MMP1 is undetectable in normal tissues and during GI inflammation is mainly secreted by myofibroblasts, macrophages, mononuclear cells, and smooth muscle cells. ${ }^{2}$ It is also secreted by epithelial cells and inflammatory cells in damaged tissues. ${ }^{32}$ It is one of the most important MMPs regulating the chemokine signaling by their complete degradation and creating receptor antagonists. ${ }^{32}$ It is known to cleave TNF $\alpha$, monocyte chemoattractant protein (MCP). ${ }^{35}$ It has been found through patient's study that MMP1 gene expression is directly associated with UC patients and if left untreated may cause the expansion of UC to small bowel. ${ }^{36}$ It has also been found to be a key marker of epithelial mesenchymal transition during progression of UC to colon cancer. ${ }^{37} \mathrm{MMP} 1$ has already been established as a blood biomarker for chronic progressive fibrotic lung disease ${ }^{38}$ and as a urinary biomarker for bladder cancer, ${ }^{39}$ supporting its stability as a secretory protein in different human body fluids; hence, we propose it as a potential candidate for fecal biomarker of UC.

\section{MMP2 (gelatinase A)}

In normal GI tract, MMP2 is secreted by epithelial cells and lamina propria of colon, fibroblasts, and immune cells, while during inflammation, it is also produced by subepithelial and pericryptal fibroblasts/myofibroblasts, mononuclear cells, epithelial cells, and vascular endothelial cells. ${ }^{2}$ Downregulation of MMP2 is found to be directly associated with endostatin and angiostatin production (the two important antiangiogenic factors), resulting in delayed wound healing in experimental UC. ${ }^{40}$ MMP2 has also been linked with cleaving of endothelin-1 to a peptide that binds to endothelin receptor signaling the upregulation of CD11/CD18 on neutrophil surfaces via mitogen-activated protein kinase pathways. This results in leukocyte adhesion and neutrophil trafficking to the inflamed tissues and initiating the healing process. ${ }^{35,41}$ The role of MMP2 as a novel, highly sensitive, diagnostic biomarker in the body fluid of patients with metastatic breast cancer has been described. ${ }^{42}$ This suggests the stability and sensitivity of MMP2 as a promising fecal biomarker for UC.

\section{MMP3 (stromelysin-I)}

In normal tissues, MMP3 is primarily expressed by fibroblasts and epithelial cells, while during inflammation in the GI tract, it is also expressed by lamina propria, stromal cells, and mononuclear macrophage cells. ${ }^{2}$ Serum levels of it were observed to be positively correlated with UC disease activity scale in children. ${ }^{43}$ It also activates multifunctional cytokine transforming growth factor $\beta$ (TGF- $\beta$ ) that regulates important cellular functions such as proliferation, apoptosis during inflammation, and wound healing. ${ }^{35,44}$ MMP3 was observed to be upregulated along with the upregulation of cytokines and chemokines in chemical-induced murine colitis model. ${ }^{45}$ It regulates chemokine signaling by facilitating the cleavage of MCP1, MCP2, MCP3, and MCP4. ${ }^{32,35}$ However cleavage of these chemokines result in an increase of associated chemokine antagonist and or inhibits leukocyte invasion by other mechanisms is not clear indicating the less pronounced anti-inflammatory effect of these products. Very recently, a study with both groups of patients affected by $\mathrm{CD}$ and $\mathrm{UC}$ showed that proteolytic degradation of $\mathrm{TNF} \alpha$-neutralizing agents such as infliximab and adalimumab by MMP3 may contribute to nonresponsiveness of patients to anti-TNF agents. ${ }^{46}$ Biomarker potential of MMP3 in body fluids like serum is reported for $\mathrm{IBD}^{46}$ and rheumatoid arthritis, ${ }^{47,48}$ and its role as a blood biomarker in other diseases such as idiopathic pulmonary fibrosis has also been reported. ${ }^{49}$ This suggests that MMP3 could also be a reliable fecal biomarker for UC.

\section{MMP7 (matrilysin)}

MMP7 is detectable in normal epithelial cells; however, during colonic inflammation, it is produced by epithelial cells and colonic mucosa. ${ }^{2,50}$ It is known to cleave TNF $\alpha$ during inflammation. ${ }^{35}$ Studies have shown that mucosal biopsy tissues of UC patients had higher MMP7 expression compared to those of $\mathrm{CD}$ patients, indicating a positive correlation between its expression and disease activity. ${ }^{27,51,52}$ It has been suggested that MMP7 contributes to intestinal wound closure but overexpression may delay epithelial wound healing. ${ }^{53,54}$ It may also aid the growth of myofibroblasts and their functions ${ }^{53}$, and stromal MMP7 may take part in maintaining mucosal homeostasis. Importance of MMP7 as a biomarker in body fluids like blood has been shown in idiopathic pulmonary fibrosis. ${ }^{38,55}$ Urinary MMP7 level, as a strong noninvasive surrogate biomarker for chronic kidney diseases, has also been reported ${ }^{56}$ due to its direct correlation with Wnt/ $\beta$-catenin signaling. Serum MMP7 is shown to be an independent prognostic factor in patients with locally advanced and/or metastatic bladder cancers ${ }^{57}$ as well as pancreatic cancer. ${ }^{58}$ Study done by Takai et al with Japanese population for screening the CRC patients showed the high sensitivity and specificity of it as a fecal marker, especially in combination with fecal cyclooxygenase2 (COX2), and 
sensitivity was as high as $90 \% .{ }^{59}$ Therefore, based on its proven stability in other secretory body fluids as well as in feces, MMP7 could be a good candidate as a fecal biomarker for UC.

\section{MMP9 (gelatinase B)}

MMP9 is undetectable in normal tissues but highly expressed by epithelial cells, immune cells, as well as neutrophils and some by macrophages during colonic inflammation. ${ }^{2}$ Among all the MMPs, MMP9 is the most divergent one for being involved with a variety of signaling molecules and pathways. MMP9 shares homology with promoter of NFאB and activator protein 1 and thereby activates proinflammatory cytokines such as IL-1 and TGF- $\beta .{ }^{35}$ MMP9 is reported to be involved in the migration of T lymphocytes, leukocyte invasion, and transendothelial migration and functions as a regulator and effector of inflammation. ${ }^{35,60,61}$ Studies have shown that MMP9 can cleave proinflammatory cytokine TNF $\alpha$ affecting its binding affinity to its receptor and manipulates inflammation. ${ }^{35,62}$ MMP9 can also cleave chemokine IL-8 in a positive feedback loop manner via chemokine receptor 1. 35,63 Other chemokines that are proteolytically cleaved by MMP9 are granulocyte chemotactic protein 2 and epithelial cellderived neutrophil-activating peptide $78 .{ }^{35,64}$ Generation and perpetuation of inflammation in UC has been reported due to dysregulated MMP9 expression and activity ${ }^{27,43,65,66}$ and its ability to cleave proinflammatory cytokines and destruct basement membrane (BM). Degradation of BM is a unique feature reflecting the severity of UC and can result into epithelial cell apoptosis, affecting normal barrier function and mucosal integrity. Damaged BM can also facilitate lymphocyte and neutrophil transmigration to the inflammatory site. ${ }^{66}$ Advantages of MMP9 as a serum biomarker in different malignancies have been reported, for example, in $\mathrm{CD},{ }^{67}$ Duchenne muscular dystrophy, ${ }^{68}$ and pulmonary tuberculosis. ${ }^{69}$ It has also been presented as a biomarker for UC as a buccal swab or in the peripheral blood for UC patients. ${ }^{27}$ MMP9 has also been successfully used as a urinary protein biomarker for bladder cancer. ${ }^{70}$ In fact, its efficacy as a fecal marker has been already displayed in pediatric $\mathrm{IBD}^{71}$ as well as while screening UC patients. ${ }^{72}$ These together implicate stability of MMP9 in different body fluids including feces. Therefore, it could be a very reliable fecal biomarker to understand the invasiveness of UC.

\section{MMPIO (stromelysin-2)}

In noninflamed tissues, MMP10 is expressed in stromal cells, while during inflammation, it is also expressed by macrophages, lymphocytes, and epithelial cells. ${ }^{2}$ The mechanism of its precise role in UC is not well understood. It has been shown that MMP10, produced by infiltrating myeloid cells, promotes mucosal healing in murine colitis. It plays a role in disease resolution by downregulating the leukocyte migration. ${ }^{73}$ In the absence of this enzyme, colonic inflammation persists and eventually results in the development of dysplastic lesions. Single nucleotide polymorphism study with UC patients displayed strong association of MMP10 expression with disease activity. ${ }^{27}$ Even though not much is known about the functionality of MMP10, it has been used as a biomarker in different malignancies. In bladder cancer, it has been shown as a urinary pharmacodynamic biomarker. ${ }^{39}$ Its efficiency as a cerebrospinal fluid has been demonstrated in Alzheimer's disease. ${ }^{74}$ Thus, MMP10 may be worth considering as a fecal biomarker.

\section{MMP I 2}

In GI tract, MMP12 is normally expressed by macrophages, while during inflammation, it can also be expressed by plump macrophage-like cells of lamina propria. ${ }^{2}$ MMP12 expression was observed in inflamed IBD mucosa when induced by the cytokine TNF $\alpha .{ }^{75}$ However, not much is known about the mechanistic pathway by which it modulates the gut inflammation. Similar to MMP3, among the patients affected by CD and $\mathrm{UC}$, it is observed to be associated with the proteolytic degradation of TNF $\alpha$-neutralizing agents such as infliximab and adalimumab, contributing to nonresponsiveness of patients to anti-TNF agents. ${ }^{46}$ However, a significant evidence of any correlation between MMP12 expression and UC is still lacking. ${ }^{27}$ Sputum levels of MMP12 and bronchioalveolar lavage fluid concentrations of MMP12 have been considered as biomarkers in a variety of lung malignancies such as chronic obstructive pulmonary disease ${ }^{76}$ and lung cancer due to chronic inflammation. ${ }^{77}$

\section{MMP I 4 (membrane type I MMP)}

MMP14 is expressed by osteoclasts in the normal tissues and myofibroblasts during inflammation. ${ }^{2}$ It is one of the important MMPs in IBD. ${ }^{2,78}$ It activates other MMPs and regulates their activity by site-specific anchoring. It also participates in maintaining levels of other MMPs either by activating or by degrading them. ${ }^{27,79} \mathrm{MMP} 14$ can also cleave proinflammatory cytokines TNF $\alpha$ and TGF- $\beta,{ }^{35}$ and thereby controls cellular inflammation and wound healing by modulating apoptosis and proliferation. It has also been reported to cleave chemokine MCP3. ${ }^{35}$ It cleaves the N-terminus of MCP3 to form its antagonist, ${ }^{80}$ although, the stability and 
efficacy of MMP14 as a biomarker in different body fluids is not evident so far. However, serum levels of MMP14 were found to be elevated among female patients with malignant ovarian tumors. ${ }^{81}$ Peripheral blood or a buccal swab suggested a positive correlation between MMP14 expression and UC activity. ${ }^{27}$ Considering MMP14 as a fecal biomarker might be naïve, but its potential cannot be ignored.

\section{Other fecal markers}

$\mathrm{UC}$, which is an inflammatory condition of colon, is associated with the migration of leucocytes to the site of inflammation and is mediated by a variety of proteins, which can be detected in human feces. In UC, chronic inflammation is perpetuated by lymphocytes and plasma cells, while neutrophil migration and degranulate substances such as myeloperoxidase (MPO), lactoferrin, and eosinophil chemotactic factors mark acute inflammation. MPO, which is a direct measure of inflammation, is a very stable enzyme secreted by activated neutrophils, and its activity correlates with cell numbers as low as 500. ${ }^{8}$ MPO levels in feces of active UC patients corroborated with endoscopic grade of inflammation and also went down with the resolution of disease. This suggests that fecal MPO is a simple, noninvasive, and efficient biomarker for UC. ${ }^{82}$ Sensitivity and efficacy of MPO to measure inflammatory activity in UC is very high compared to other similar fecal markers, such as eosinophilic cationic protein and eosinophil peroxidase granule. ${ }^{83}$ Lactoferrin, an iron binding protein, secreted by intestinal mucosa and other body fluids is another fecal marker to measure disease activity and was observed to have $90 \%$ specificity with UC patients. ${ }^{8,84-86}$ Calprotectin, which is another common fecal biomarker to assess disease activity in UC patients, is a calcium-binding protein that inhibits MMPs. ${ }^{61}$ Although studies have shown that it can differentiate between UC and irritable bowel syndrome, it is used as a complementary test because its fecal levels are affected by intake of nonsteroidal anti-inflammatory drug, bleeding volume, and malignancy. ${ }^{8}$ Ahmed et al also suggested fecal miRNA as a promising sensitive and specific biomarker for UC screening; ${ }^{87}$ however, reliability of it is still questionable due to the presence of relatively abundant class of transcripts and tissues, specifically neutrophil gelatinase-associated lipocalin and its murine homolog. Lipocalin 2 (Lcn2) belongs to a family of small, secreted proteins expressed by a variety of cells, the richest source being neutrophils. Both fecal neutrophil gelatinase-associated lipocalin (in a clinical study) and fecal Lcn2 (in murine model of colitis) were observed to be a sensitive and noninvasive biomarker of colonic inflammation. ${ }^{88,89}$ Interestingly, fecal markers are also finding their way as biomarkers for CRC. Patients with UC are more susceptible to CRC. Takai et al have shown that a combination of fecal COX2 (an enzyme expressed only during inflammation) along with MMP7 had 100\% specificity in CRC screening. ${ }^{59}$

\section{Limitations}

Past studies have shown a weak correlation of fecal biomarkers and clinical activity indices for CD while a better correlation for UC and have emphasized on including the endoscopic and histological assessments into consideration. ${ }^{90}$ The main disadvantage of fecal biomarker is the "positive predictive value", which means a positive fecal biomarker test is less specific or indicative of the disease advancement. ${ }^{91}$ A positive value can correlate with colonic inflammation or lesions but is difficult to predict that it is due to UC only. Patients under the treatment can also have false-positive result of MMP activation due to antibiotic or nonsteroidal anti-inflammatory drug treatments. Thus, a positive test result will probably prompt further evaluation, while a negative test would imply that there is no inflammation, in which case additional anti-inflammatory drugs could be avoided. Therefore, fecal biomarkers can substitute invasive, expensive, time consuming, and not always available "endoscopy procedure", which is often not very well accepted by patients (especially, among children due to fasting and bowel preparation) $)^{90}$ but cannot replace the endoscopies completely. In modern UC treatment, mucosal healing is the primary therapeutic target, despite the existence of clinically valid definition for it, which creates another limitation for the use of MMPs as fecal biomarkers due to the fact that some MMPs (Figure 1) such as MMP2, MMP3, MMP7, and MMP12 are expressed by normal tissues. Other than the difference between their quantitative expression levels in normal versus inflamed UC tissues, sources of the cells that express these MMPs are also critical. For example, in normal tissues, MMP2 is secreted by macrophage mainly, while it is expressed by epithelial cells, macrophages, as well as dendritic cells (Figure 1) during UC. Therefore, it will be hard to conclude that elevated levels of it during the healing phase is a true picture of healing or failure of the therapy, unless it is supported by histology or endoscopy or fecal specimen of the patient has been examined at regular intervals. This implies the importance of either repetitive endoscopic examinations or necessity of the use of surrogate markers. ${ }^{90}$ Hence, fecal biomarkers, which are undoubtedly an emerging field with a lot of promise and 


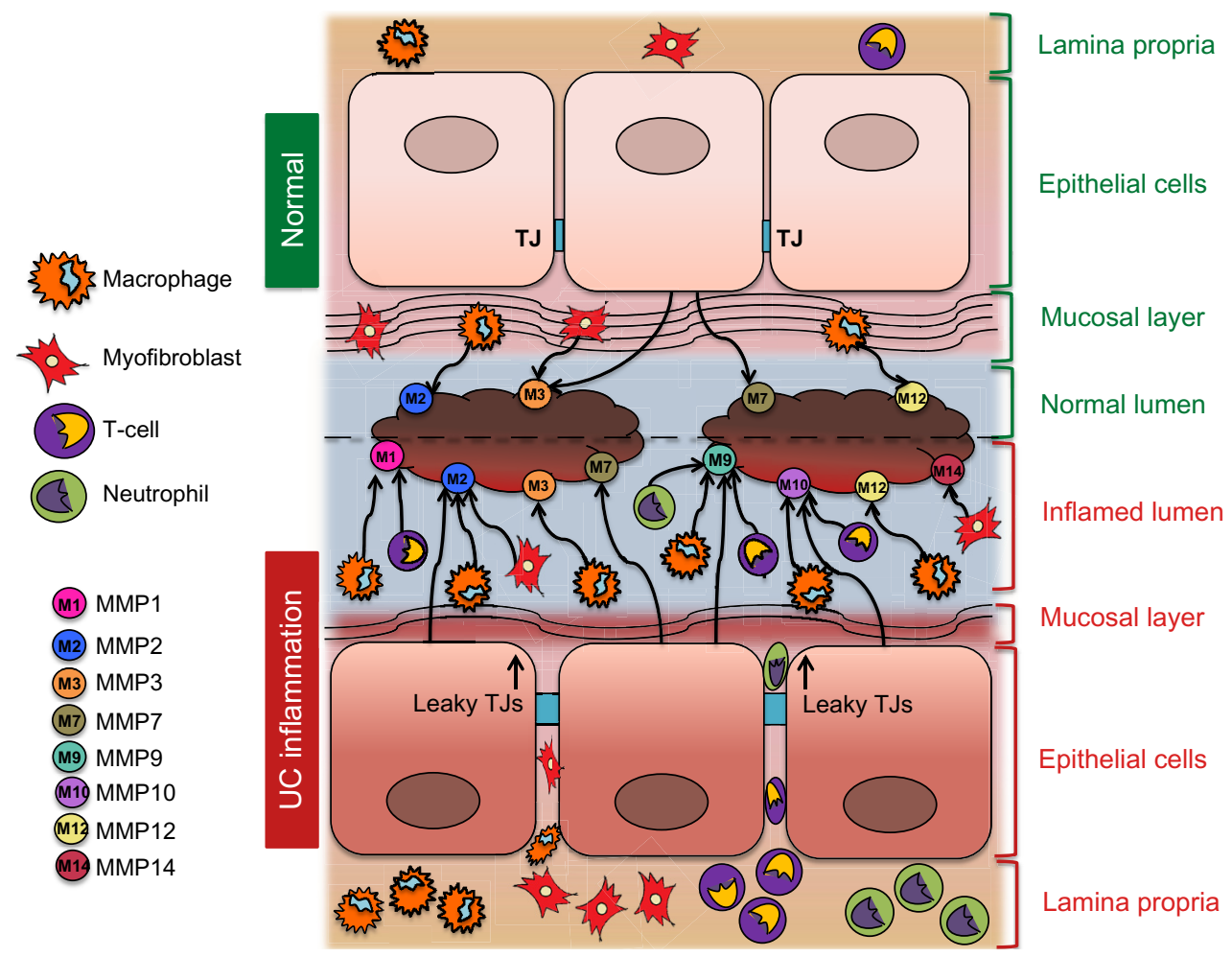

Figure I A schematic presentation of MMPs active during UC and in the normal colonic tissue.

Notes: The schematic shows immune cells (neutrophils, T-cells, macrophages), myofibroblasts, and epithelial cells as the sources of different MMPs active during UC and in the normal colonic tissue. It indicates an increase in the infiltration of immune cells and myofibroblasts through the epithelial-mucosal barrier, via leaky tight junctions during UC. Abbreviations: MMP, matrix metalloproteinase; UC, ulcerative colitis; TJ, tight junction; M, MMP.

ease, need more validation for their success. Therefore, in the present scenario, fecal markers could successfully be used as an assessment tool for the treatment success and as a monitoring tool during UC therapy.

\section{Conclusion}

Indisputably, fecal biomarkers represent the new and specific class of biomarkers for the early detection of UC, allowing the easy assessment of disease status and personalized therapeutic interventions for individual patients. We introduce MMPs that are secreted by a variety of cells during inflammation or healing phases and play an active role in mucositis as the most promising fecal biomarker (Figure 1). Table 1 displays their stability in different human body fluids as well as their recognition as efficient biomarkers to evaluate different malignancies. A comprehensive assessment of literature recommends that specificity and sensitivity of MMPs compared to other fecal biomarkers are very high, especially for MMP9 in UC. Clinical studies have also shown that fecal MMP9 is a reliable marker to understand UC activity. The most striking feature of MMP9 is that it is secreted by the inflamed cells only, not by normal healthy tissues, and is hence undetectable in normal subjects. MMP7 could have been another plausible candidate as a fecal biomarker. Its credibility as a biomarker has been well established in different body fluids for a variety of diseases. However, one concern with MMP7 is its presence in normal tissues, which might give a false-positive value unless the screening is performed at regular intervals for its validation. On the other hand, a trace of MMP9 would correlate well with the onset of UC. Furthermore, MMP9 has also proven to be more specific and sensitive to differentiate between CD and UC patients. Therefore, among MMPs as a fecal biomarker, MMP9 seems to be the most preferred one. Further, we suggest the initial screening of MMPs in feces as UC biomarkers as a noninvasive strategy for the clinical care of the patients. As discussed in the "Limitations" section, the only drawback with this approach is the nonavailability of established inflammatory score or grading with MMPs levels in feces. However, this could be resolved by performing routine colonoscopy only for those patients reflecting significant levels of MMPs to identify the inflamed areas and further monitoring to avoid development of colon cancer. Therefore, future studies correlating inflammatory score with MMP levels are needed to completely avoid invasive screening by colonoscopy. Using MMPs as fecal biomarkers, healing after 
Table I The comparative analysis of MMPs (also active in UC) as different body-fluid biomarkers

\begin{tabular}{|c|c|c|c|c|c|c|}
\hline \multirow[t]{2}{*}{ Name of MMPs } & \multirow[t]{2}{*}{ Mechanistic role in intestinal inflammation } & \multicolumn{5}{|c|}{$\begin{array}{l}\text { Established biomarker activity in different } \\
\text { malignancies }\end{array}$} \\
\hline & & Serum & Urine & Blood & Feces & CSF \\
\hline MMPI (collagenase I) & Cleaves TNF $\alpha$, MCP. & - & $++^{39}$ & $+^{38}$ & - & - \\
\hline MMP2 (gelatinase A) & $\begin{array}{l}\text { Cleaves endothelin-I to a peptide that binds to } \\
\text { endothelin receptor signaling the upregulation of } \\
\text { CDII/CDI } 8 \text { on neutrophil surfaces via MAPK } \\
\text { pathways. } \\
\text { Triggers the leukocyte adhesion and neutrophil } \\
\text { trafficking to inflamed tissues. }\end{array}$ & - & - & - & - & - \\
\hline MMP3 (stromelysin-I) & $\begin{array}{l}\text { Activates TGF- } \beta \text {. } \\
\text { Regulates chemokine signaling by facilitating the } \\
\text { cleavage of MCPI, MCP2, MCP3, and MCP4. } \\
\text { Inhibition of leukocyte invasion is unknown, indicating } \\
\text { the less pronounced anti-inflammatory effect. }\end{array}$ & $++^{43}$ & - & $++^{49}$ & - & - \\
\hline MMP7 (matrilysin) & $\begin{array}{l}\text { Cleaves TNF } \alpha \text {. } \\
\text { Aids the growth of myofibroblasts that may take part } \\
\text { in maintaining the mucosal homeostasis. } \\
\text { Overexpression may delay epithelial wound healing. }\end{array}$ & $+^{57}$ & $+^{56}$ & $++^{48,55}$ & $++^{59}$ & - \\
\hline MMP9 (gelatinase B) & $\begin{array}{l}\text { Activates proinflammatory cytokines such as IL-I, } \\
\text { TGF- } \beta \text {, and TNF } \alpha \text {. } \\
\text { Involved with the migration of T lymphocytes, } \\
\text { leukocyte invasion, and transendothelial migration } \\
\text { to regulate inflammation. } \\
\text { Cleaves chemokines such as IL-8, GCP-2, and ENA-78. } \\
\text { Degrades BM facilitating lymphocytes and neutrophil } \\
\text { migration to the inflammatory site. }\end{array}$ & $+^{67-69}$ & $+^{70}$ & $++^{27}$ & $++^{71}$ & - \\
\hline MMPIO (stromelysin-2) & Mechanism not yet known. & - & $++^{39}$ & - & - & $+^{73}$ \\
\hline MMPI2 & Mechanism not yet known. & - & - & - & - & - \\
\hline $\begin{array}{l}\text { MMPI4 (membrane } \\
\text { type I MMP) }\end{array}$ & Cleaves proinflammatory cytokines TNF $\alpha$ and TGF- $\beta$. & $++^{80}$ & - & $+{ }^{27}$ & - & - \\
\hline
\end{tabular}

Abbreviations: MMP, matrix metalloproteinase; UC, ulcerative colitis; CSF, cerebrospinal fluid; TNF $\alpha$, tumor necrosis factor alpha; MCP, monocyte chemoattractant protein; MAPK, mitogen-activated protein kinase; TGF- $\beta$, transforming growth factor $\beta$; IL-I, interleukin-I; IL-8, interleukin-8; GCP-2, granulocyte chemotactic protein 2; ENA-78, epithelial cell-derived neutrophil-activating peptide 78; BM, basement membrane; -, not known or not available.

treatment can also be monitored in a noninvasive manner. This would be advantageous in determining relapses and can avert repeated colonoscopy.

\section{Disclosure}

The authors report no conflicts of interest in this work.

\section{References}

1. Giannandrea M, Parks WC. Diverse functions of matrix metalloproteinases during fibrosis. Dis Model Mech. 2014;7(2):193-203.

2. Ravi A, Garg P, Sitaraman SV. Matrix metalloproteinases in inflammatory bowel disease: boon or a bane? Inflamm Bowel Dis. 2007;13(1):97-107.

3. O'Sullivan S, Gilmer JF, Medina C. Matrix metalloproteinases in inflammatory bowel disease: an update. Mediators Inflamm. 2015;2015:964131.

4. Rodriguez D, Morrison CJ, Overall CM. Matrix metalloproteinases: what do they not do? New substrates and biological roles identified by murine models and proteomics. Biochim Biophys Acta. 2010;1803(1): $39-54$.

5. Iskandar HN, Ciorba MA. Biomarkers in inflammatory bowel disease: current practices and recent advances. Transl Res. 2012;159(4):313-325.
6. Mackalski BA, Bernstein CN. New diagnostic imaging tools for inflammatory bowel disease. Gut. 2006;55(5):733-741.

7. Rameshshanker R, Arebi N. Endoscopy in inflammatory bowel disease when and why. World J Gastrointest Endosc. 2012;4(6):201-211.

8. Masoodi I, Tijjani BM, Wani H, Hassan NS, Khan AB, Hussain S. Biomarkers in the management of ulcerative colitis: a brief review. Ger Med Sci. 2011;9:Doc03.

9. Talley NJ, Abreu MT, Achkar JP, et al; American College of Gastroenterology IBD Task Force. An evidence-based systematic review on medical therapies for inflammatory bowel disease. Am J Gastroenterol. 2011;106(suppl 1):S2-S25; quiz S26.

10. Tozer PJ, Whelan K, Phillips RK, Hart AL. Etiology of perianal Crohn's disease: role of genetic, microbiological, and immunological factors. Inflamm Bowel Dis. 2009;15(10):1591-1598.

11. Li C, Kuemmerle JF. Mechanisms that mediate the development of fibrosis in patients with Crohn's disease. Inflamm Bowel Dis. 2014;20(7):1250-1258.

12. Geller SA, de Campos FP. Crohn disease. Autops Case Rep. 2015;5(2):5-8.

13. Zemljic M, Pejkovic B, Krajnc I, Lipovsek S. Biological pathways involved in the development of inflammatory bowel disease. Wien Klin Wochenschr. 2014;126(19-20):626-633.

14. Walter L, Harper C, Garg P. Role of matrix metalloproteinases in inflammation/colitis-associated colon cancer. Immunogastroenterology. 2013;2:22-28. 
15. Silosi I, Boldeanu MV, Mogoanta SS, et al. Matrix metalloproteinases (MMP-3 and MMP-9) implication in the pathogenesis of inflammatory bowel disease (IBD). Rom J Morphol Embryol. 2014;55(4): 1317-1324.

16. Meijer MJ, Mieremet-Ooms MA, van der Zon AM, et al. Increased mucosal matrix metalloproteinase-1, $-2,-3$ and -9 activity in patients with inflammatory bowel disease and the relation with Crohn's disease phenotype. Dig Liver Dis. 2007;39(8):733-739.

17. Gao Q, Meijer MJ, Kubben FJ, et al. Expression of matrix metalloproteinases-2 and -9 in intestinal tissue of patients with inflammatory bowel diseases. Dig Liver Dis. 2005;37(8):584-592.

18. Matusiewicz M, Neubauer K, Mierzchala-Pasierb M, Gamian A, Krzystek-Korpacka M. Matrix metalloproteinase-9: its interplay with angiogenic factors in inflammatory bowel diseases. Dis Markers. 2014;2014:643-645.

19. Langers AM, Verspaget HW, Hawinkels LJ, et al. MMP-2 and MMP-9 in normal mucosa are independently associated with outcome of colorectal cancer patients. Br J Cancer. 2012;106(9):1495-1498.

20. Palli D, Trallori G, Saieva C, et al. General and cancer specific mortality of a population based cohort of patients with inflammatory bowel disease: the Florence Study. Gut. 1998;42(2):175-179.

21. Winther KV, Jess T, Langholz E, Munkholm P, Binder V. Survival and cause-specific mortality in ulcerative colitis: follow-up of a population-based cohort in Copenhagen County. Gastroenterology. 2003;125(6):1576-1582.

22. Cosnes J, Gower-Rousseau C, Seksik P, Cortot A. Epidemiology and natural history of inflammatory bowel diseases. Gastroenterology. 2011;140(6):1785-1794.

23. Abou El Azm AR, Yousef M, Kobtan A, Awad A, Elkassas G, Elfert A Colonic mucosal expression of heat-shock proteins may have a potential prognostic value in ulcerative colitis. Arab J Gastroenterol. 2015;16(1):20-24.

24. Wallace KL, Zheng LB, Kanazawa Y, Shih DQ. Immunopathology of inflammatory bowel disease. World J Gastroenterol. 2014;20(1): 6-21.

25. Koboziev I, Reinoso Webb C, Furr KL, Grisham MB. Role of the enteric microbiota in intestinal homeostasis and inflammation. Free Radic Biol Med. 2014;68:122-133.

26. Overall CM. Molecular determinants of metalloproteinase substrate specificity: matrix metalloproteinase substrate binding domains, modules, and exosites. Mol Biotechnol. 2002;22(1):51-86.

27. Morgan AR, Han DY, Lam WJ, et al. Genetic variations in matrix metalloproteinases may be associated with increased risk of ulcerative colitis. Hum Immunol. 2011;72(11):1117-1127.

28. Matrisian LM. Metalloproteinases and their inhibitors in matrix remodeling. Trends Genet. 1990;6(4):121-125.

29. Kessenbrock K, Plaks V, Werb Z. Matrix metalloproteinases: regulators of the tumor microenvironment. Cell. 2010;141(1):52-67.

30. Castaneda FE, Walia B, Vijay-Kumar M, et al. Targeted deletion of metalloproteinase 9 attenuates experimental colitis in mice: central role of epithelial-derived MMP. Gastroenterology. 2005;129(6):1991-2008.

31. Garg P, Vijay-Kumar M, Wang L, Gewirtz AT, Merlin D, Sitaraman SV. Matrix metalloproteinase-9-mediated tissue injury overrides the protective effect of matrix metalloproteinase-2 during colitis. Am J Physiol Gastrointest Liver Physiol. 2009;296(2):G175-G184.

32. Gill SE, Parks WC. Metalloproteinases and their inhibitors: regulators of wound healing. Int J Biochem Cell Biol. 2008;40(6-7):1334-1347.

33. Vincent C, Mehrotra S, Loo VG, Dewar K, Manges AR. Excretion of host DNA in feces is associated with risk of Clostridium difficile infection. J Immunol Res. 2015;2015:246203.

34. Sugi K, Saitoh O, Hirata I, Katsu K. Fecal lactoferrin as a marker for disease activity in inflammatory bowel disease: comparison with other neutrophil-derived proteins. Am J Gastroenterol. 1996;91(5): 927-934.

35. Le NT, Xue M, Castelnoble LA, Jackson CJ. The dual personalities of matrix metalloproteinases in inflammation. Front Biosci. 2007; $12: 1475-1487$.
36. Yanai H, Ben-Shachar S, Baram L, et al. Gene expression alterations in ulcerative colitis patients after restorative proctocolectomy extend to the small bowel proximal to the pouch. Gut. 2015;64(5):756-764.

37. Zhao X, Fan J, Zhi F, et al. Mobilization of epithelial mesenchymal transition genes distinguishes active from inactive lesional tissue in patients with ulcerative colitis. Hum Mol Genet. 2015;24(16):4615-4624.

38. Rosas IO, Richards TJ, Konishi K, et al. MMP1 and MMP7 as potential peripheral blood biomarkers in idiopathic pulmonary fibrosis. PLoS Med. 2008;5(4):e93.

39. Du X, Lin BC, Wang QR, et al. MMP-1 and Pro-MMP-10 as potential urinary pharmacodynamic biomarkers of FGFR3-targeted therapy in patients with bladder cancer. Clin Cancer Res. 2014;20(24): 6324-6335.

40. Deng X, Tolstanova G, Khomenko T, et al. Mesalamine restores angiogenic balance in experimental ulcerative colitis by reducing expression of endostatin and angiostatin: novel molecular mechanism for therapeutic action of mesalamine. J Pharmacol Exp Ther. 2009; 331(3):1071-1078.

41. Fernandez-Patron C, Zouki C, Whittal R, Chan JS, Davidge ST, Filep JG. Matrix metalloproteinases regulate neutrophil-endothelial cell adhesion through generation of endothelin-1[1-32]. FASEB J. 2001;15(12):2230-2240.

42. Noh S, Jung JJ, Jung M, et al. Body fluid MMP-2 as a putative biomarker in metastatic breast cancer. Oncol Lett. 2012;3(3):699-703.

43. Kofla-Dlubacz A, Matusiewicz M, Krzesiek E, Noga L, Iwańczak B. Metalloproteinase-3 and -9 as novel markers in the evaluation of ulcerative colitis activity in children. Adv Clin Exp Med. 2014;23(1):103-110.

44. Maeda S, Dean DD, Gomez R, Schwartz Z, Boyan BD. The first stage of transforming growth factor betal activation is release of the large latent complex from the extracellular matrix of growth plate chondrocytes by matrix vesicle stromelysin-1 (MMP-3). Calcif Tissue Int. 2002;70(1):54-65

45. te Velde AA, de Kort F, Sterrenburg E, et al. Comparative analysis of colonic gene expression of three experimental colitis models mimicking inflammatory bowel disease. Inflamm Bowel Dis. 2007; 13(3):325-330.

46. Biancheri P, Brezski RJ, Di Sabatino A, et al. Proteolytic cleavage and loss of function of biologic agents that neutralize tumor necrosis factor in the mucosa of patients with inflammatory bowel disease. Gastroenterology. 2015;149(6):1564.e-1574.e.

47. Centola M, Cavet G, Shen Y, et al. Development of a multibiomarker disease activity test for rheumatoid arthritis. PLoS One. 2013;8(4):e60635.

48. Mease PJ. The potential roles for novel biomarkers in rheumatoid arthritis assessment. Clin Exp Rheumatol. 2011;29(3):567-574.

49. DePianto DJ, Chandriani S, Abbas AR, et al. Heterogeneous gene expression signatures correspond to distinct lung pathologies and biomarkers of disease severity in idiopathic pulmonary fibrosis. Thorax. 2015;70(1):48-56.

50. Rath T, Roderfeld M, Halwe JM, Tschuschner A, Roeb E, Graf J. Cellular sources of MMP-7, MMP-13 and MMP-28 in ulcerative colitis. Scand J Gastroenterol. 2010;45(10):1186-1196.

51. Bjerrum JT, Nyberg C, Olsen J, Nielsen OH. Assessment of the validity of a multigene analysis in the diagnostics of inflammatory bowel disease. J Intern Med. 2014;275(5):484-493.

52. Matsuno K, Adachi Y, Yamamoto H, et al. The expression of matrix metalloproteinase matrilysin indicates the degree of inflammation in ulcerative colitis. J Gastroenterol. 2003;38(4):348-354.

53. Makitalo L, Piekkala M, Ashorn M, et al. Matrix metalloproteinases in the restorative proctocolectomy pouch of pediatric ulcerative colitis. World J Gastroenterol. 2012;18(30):4028-4036.

54. Hayden DM, Forsyth C, Keshavarzian A. The role of matrix metalloproteinases in intestinal epithelial wound healing during normal and inflammatory states. J Surg Res. 2011;168(2):315-324.

55. Song JW, Do KH, Jang SJ, Colby TV, Han S, Kim DS. Blood biomarkers MMP-7 and SP-A: predictors of outcome in idiopathic pulmonary fibrosis. Chest. 2013;143(5):1422-1429. 
56. He W, Tan RJ, Li Y, et al. Matrix metalloproteinase-7 as a surrogate marker predicts renal Wnt/beta-catenin activity in CKD. J Am Soc Nephrol. 2012;23(2):294-304.

57. Szarvas T, Becker M, vom Dorp F, et al. Matrix metalloproteinase-7 as a marker of metastasis and predictor of poor survival in bladder cancer. Cancer Sci. 2010;101(5):1300-1308.

58. Fukuda A, Wang SC, Morris JP, et al. Stat3 and MMP7 contribute to pancreatic ductal adenocarcinoma initiation and progression. Cancer Cell. 2011;19(4):441-455.

59. Takai T, Kanaoka S, Yoshida K, et al. Fecal cyclooxygenase 2 plus matrix metalloproteinase 7 mRNA assays as a marker for colorectal cancer screening. Cancer Epidemiol Biomarkers Prev. 2009; 18(6): 1888-1893.

60. Leppert D, Waubant E, Galardy R, Bunnett NW, Hauser SL. T cell gelatinases mediate basement membrane transmigration in vitro. J Immunol. 1995;154(9):4379-4389.

61. Allport JR, Lim YC, Shipley JM, et al. Neutrophils from MMP-9- or neutrophil elastase-deficient mice show no defect in transendothelial migration under flow in vitro. $J$ Leukoc Biol. 2002;71(5):821-828.

62. Gearing AJ, Beckett P, Christodoulou M, et al. Processing of tumour necrosis factor-alpha precursor by metalloproteinases. Nature. 1994;370(6490):555-557.

63. Van den Steen PE, Proost P, Wuyts A, Van Damme J, Opdenakker G. Neutrophil gelatinase B potentiates interleukin- 8 tenfold by aminoterminal processing, whereas it degrades CTAP-III, PF-4, and GROalpha and leaves RANTES and MCP-2 intact. Blood. 2000;96(8): 2673-2681.

64. Van Den Steen PE, Wuyts A, Husson SJ, Proost P, Van Damme J, Opdenakker G. Gelatinase B/MMP-9 and neutrophil collagenase/ MMP-8 process the chemokines human GCP-2/CXCL6, ENA-78/ CXCL5 and mouse GCP-2/LIX and modulate their physiological activities. Eur J Biochem. 2003;270(18):3739-3749.

65. Marshall DC, Lyman SK, McCauley S, et al. Selective allosteric inhibition of MMP9 is efficacious in preclinical models of ulcerative colitis and colorectal cancer. PLoS One. 2015;10(5):e0127063.

66. Lakatos G, Sipos F, Miheller P, et al. The behavior of matrix metalloproteinase-9 in lymphocytic colitis, collagenous colitis and ulcerative colitis. Pathol Oncol Res. 2012;18(1):85-91.

67. Faubion WA Jr, Fletcher JG, O’Byrne S, et al. EMerging BiomARKers in Inflammatory Bowel Disease (EMBARK) study identifies fecal calprotectin, serum MMP9, and serum IL-22 as a novel combination of biomarkers for Crohn's disease activity: role of cross-sectional imaging. Am J Gastroenterol. 2013;108(12):1891-1900.

68. Nadarajah VD, van Putten M, Chaouch A, et al. Serum matrix metalloproteinase-9 (MMP-9) as a biomarker for monitoring disease progression in Duchenne muscular dystrophy (DMD). Neuromuscul Disord. 2011;21(8):569-578.

69. Xu D, LiY, Li X, et al. Serum protein S100A9, SOD3, and MMP9 as new diagnostic biomarkers for pulmonary tuberculosis by iTRAQ-coupled two-dimensional LC-MS/MS. Proteomics. 2015;15(1):58-67.

70. Rosser CJ, Chang M, Dai Y, et al. Urinary protein biomarker panel for the detection of recurrent bladder cancer. Cancer Epidemiol Biomarkers Prev. 2014;23(7):1340-1345.

71. Kolho KL, Sipponen T, Valtonen E, Savilahti E. Fecal calprotectin, MMP-9, and human beta-defensin-2 levels in pediatric inflammatory bowel disease. Int J Colorectal Dis. 2014;29(1):43-50.

72. Annahazi A, Molnar T, Farkas K, et al. Fecal MMP-9: a new noninvasive differential diagnostic and activity marker in ulcerative colitis. Inflamm Bowel Dis. 2013;19(2):316-320.

73. Koller FL, Dozier EA, Nam KT, et al. Lack of MMP10 exacerbates experimental colitis and promotes development of inflammationassociated colonic dysplasia. Lab Invest. 2012;92(12):1749-1759.

74. Craig-Schapiro R, Kuhn M, Xiong C, et al. Multiplexed immunoassay panel identifies novel CSF biomarkers for Alzheimer's disease diagnosis and prognosis. PLoS One. 2011;6(4):e18850.
75. Pedersen G, Saermark T, Kirkegaard T, Brynskov J. Spontaneous and cytokine induced expression and activity of matrix metalloproteinases in human colonic epithelium. Clin Exp Immunol. 2009;155(2):257-265.

76. LaPan P, Brady J, Grierson C, et al. Optimization of total protein and activity assays for the detection of MMP-12 in induced human sputum. BMC Pulm Med. 2010;10:40.

77. Qu P, Du H, Wang X, Yan C. Matrix metalloproteinase 12 overexpression in lung epithelial cells plays a key role in emphysema to lung bronchioalveolar adenocarcinoma transition. Cancer Res. 2009;69(18):7252-7261.

78. von Lampe B, Barthel B, Coupland SE, Riecken EO, Rosewicz S. Differential expression of matrix metalloproteinases and their tissue inhibitors in colon mucosa of patients with inflammatory bowel disease. Gut. 2000;47(1):63-73.

79. Strongin AY, Collier I, Bannikov G, Marmer BL, Grant GA, Goldberg GI. Mechanism of cell surface activation of $72-\mathrm{kDa}$ type IV collagenase. Isolation of the activated form of the membrane metalloprotease. J Biol Chem. 1995;270(10):5331-5338.

80. McQuibban GA, Gong JH, Wong JP, Wallace JL, Clark-Lewis I, Overall CM. Matrix metalloproteinase processing of monocyte chemoattractant proteins generates $\mathrm{CC}$ chemokine receptor antagonists with anti-inflammatory properties in vivo. Blood. 2002;100(4): $1160-1167$.

81. Kaimal R, Aljumaily R, Tressel SL, et al. Selective blockade of matrix metalloprotease-14 with a monoclonal antibody abrogates invasion, angiogenesis, and tumor growth in ovarian cancer. Cancer Res. 2013;73(8):2457-2467.

82. Saiki T. Myeloperoxidase concentrations in the stool as a new parameter of inflammatory bowel disease. Kurume Med J. 1998;45(1):69-73.

83. Sangfelt P, Carlson M, Thorn M, Lööf L, Raab Y. Neutrophil and eosinophil granule proteins as markers of response to local prednisolone treatment in distal ulcerative colitis and proctitis. Am J Gastroenterol. 2001;96(4):1085-1090.

84. Uchida K, Matsuse R, Tomita S, Sugi K, Saitoh O, Ohshiba S. Immunochemical detection of human lactoferrin in feces as a new marker for inflammatory gastrointestinal disorders and colon cancer. Clin Biochem. 1994;27(4):259-264.

85. Langhorst J, Elsenbruch S, Mueller T, et al. Comparison of 4 neutrophilderived proteins in feces as indicators of disease activity in ulcerative colitis. Inflamm Bowel Dis. 2005;11(12):1085-1091.

86. Kane SV, Sandborn WJ, Rufo PA, et al. Fecal lactoferrin is a sensitive and specific marker in identifying intestinal inflammation. Am J Gastroenterol. 2003;98(6):1309-1314.

87. Ahmed FE, Jeffries CD, Vos PW, et al. Diagnostic microRNA markers for screening sporadic human colon cancer and active ulcerative colitis in stool and tissue. Cancer Genomics Proteomics. 2009;6(5): 281-295.

88. Chassaing B, Srinivasan G, Delgado MA, Young AN, Gewirtz AT, Vijay-Kumar M. Fecal lipocalin 2, a sensitive and broadly dynamic non-invasive biomarker for intestinal inflammation. PLoS One. 2012;7(9):e44328.

89. Nielsen $\mathrm{OH}$, Gionchetti P, Ainsworth M, et al. Rectal dialysate and fecal concentrations of neutrophil gelatinase-associated lipocalin, interleukin-8, and tumor necrosis factor-alpha in ulcerative colitis. Am J Gastroenterol. 1999;94(10):2923-2928.

90. Lehmann FS, Burri E, Beglinger C. The role and utility of faecal markers in inflammatory bowel disease. Therap Adv Gastroenterol. 2015;8(1):23-36.

91. Assche GV. Fecal biomarkers for the diagnosis and management of inflammatory bowel disease. Gastroenterol Hepatol. 2011;7(6):396-398. 
Metalloproteinases In Medicine

\section{Publish your work in this journal}

Metalloproteinases In Medicine is an international, peer reviewed, open access journal that aims to provide a platform for the discussion and dissemination of knowledge about the role that metalloproteinases - such as matrix metalloproteinases (MMP), ADAMs, ADAMTSs, and astacins, as well as their inhibitors - play in diseases.

Submit your manuscript here: http://www.dovepress.com/metalloproteinases-in-medicine-journal 